\title{
Editorial: An Omics Perspective on Fungal Infection: Toward Next-Generation Diagnosis and Therapy
}

\author{
Agostinho Carvalho ${ }^{1,2 *}$ and Gustavo H. Goldman ${ }^{3 *}$ \\ ${ }^{1}$ Life and Health Sciences Research Institute (ICVS), School of Medicine, University of Minho, Braga, Portugal, ${ }^{2}$ ICVS/3B's - \\ PT Government Associate Laboratory, Braga/Guimarães, Portugal, ${ }^{3}$ Faculdade de Ciências Farmacêticas de Ribeirão \\ Preto, Universidade de São Paulo, São Paulo, Brazil
}

Keywords: omics techniques, fungal infection, personalized healthcare, antifungal therapeutics, biomarker discovery

\section{Editorial on the Research Topic}

\section{An Omics Perspective on Fungal Infection: Toward Next-Generation Diagnosis and Therapy}

OPEN ACCESS

Edited by:

Martin G. Klotz,

Queens College, City University of New York, USA

Reviewed by: Bernhard Hube,

Hans Knöll Institute, Germany

*Correspondence:

Agostinho Carvalho agostinhocarvalho@med.uminho.pt

Gustavo H. Goldman ggoldman@usp.br

Specialty section: This article was submitted to Fungi and Their Interactions, a section of the journal

Frontiers in Microbiology

Received: 19 August 2016 Accepted: 12 January 2017 Published: 26 January 2017

Citation:

Carvalho A and Goldman GH (2017) Editorial: An Omics Perspective on

Fungal Infection: Toward Next-Generation Diagnosis and Therapy. Front. Microbiol. 8:85. doi: 10.3389/fmicb.2017.00085
Fungal infections are estimated to occur in over a billion people each year, and evidence suggests the rate is increasing. Vaccines are unavailable, and despite progress in diagnosis and therapy, the management of fungal infections is a challenging endeavor associated with unacceptable mortality rates, particularly in immunocompromised hosts (Kontoyiannis et al., 2010; Pagano et al., 2010). Importantly, the risk of infection and its clinical outcome vary significantly even among patients with similar predisposing conditions (Carvalho et al., 2010). Concerns over excessive prescription of antifungals and the remarkable burden conveyed to healthcare systems have encouraged efforts to decipher the molecular and cellular causes underlying variable susceptibility to infection (Cunha and Carvalho, 2012).

The combination of omics technologies and advanced computational methods, together with the use of both established and alternative in vitro and in vivo models of infection (Brunke et al., 2015), provides comprehensive views of the architecture and dynamics of host-fungus interaction networks at a level of complexity previously unanticipated. As a result of our deepened understanding of the biological, biochemical and biophysical molecular processes regulating the host-fungus interaction, several targets with potential usefulness in personalized medical interventions have been proposed (Oliveira-Coelho et al., 2015).

The present Research Topic brings together 10 articles covering multiple aspects of the hostfungus interaction with emphasis on the application of omics-based technologies to project novel or improve current diagnostic and therapeutic approaches. Smeekens et al. point out the advent of omics platforms and the development of systems biology tools to study antifungal immunity (Smeekens et al.). Much research has been performed on host genetics and fungal infection (Cunha et al., 2013; Smeekens et al., 2013b), but only recently have these data been integrated into functional genomics approaches driving unbiased identification and quantification of targets controlling susceptibility to infection (Smeekens et al., 2013a; Kumar et al., 2014). With the increasing number and quality of data repositories, the generation of multi-scale host-fungus interaction models through systems biology is expected to support personalized medicine interventions (Dix et al., 2016).

A substantial proportion of genetic markers associated with the risk of fungal infection are within immune-related genes (Lupianez et al., 2015), often implicated in the nuclear factor (NF)-кB 
signaling pathways. Through a systematic evaluation of single nucleotide polymorphisms (SNPs) in NF- $\kappa$ B pathways, Lupiañez et al. exclude a clinically relevant impact of SNPs in these genes to the risk of invasive aspergillosis (IA) (Lupiañez et al.), thus supporting the concept that innate immune receptors, rather than molecules involved in downstream signaling, are major repositories of genetic variability regulating antifungal immune function (Bochud et al., 2008; Carvalho et al., 2008, 2012b; Cunha et al., 2010, 2014, 2015; Wojtowicz et al., 2015). As host damage perception is fundamental for resolution of infection (Cunha et al., 2012), SNPs underlying the hyperactivation of the S100 calcium-binding protein B (S100B)/receptor for advanced glycation end products danger signaling pathway have also been put forward as intrinsic factors influencing the risk for IA (Cunha et al., 2011). Accordingly, Dix et al. describe an enrichment of gene expression profiles of patients suffering from IA in the S100B transcript, therefore highlighting its potential as a valuable prognosis biomarker (Dix et al.).

The use of transcriptomics and epigenomics has also contributed to the identification and characterization of dynamic cellular processes with unparalleled resolution. An emerging view is that immune cells are able to adapt their metabolic programs to meet specialized defense needs through the precise and concerted action of epigenetic mechanisms and metabolic pathways (Cheng et al., 2014; Saeed et al., 2014). Hellwig et al. provide evidence that the effector functions of natural killer $\mathrm{T}$ cells in response to Candida albicans are also critically dependent on metabolic plasticity (Hellwig et al.). This finding is in line with the metabolic reprogramming and epigenetic imprinting occurring in monocytes in response to $\beta$-glucan (Netea et al., 2016). Understanding how metabolism coordinates immune cell function might uncover innovative therapeutics or metabolic adjuncts to reorient cells toward immune protection (Cheng et al., 2016).

The adaptation of the fungus to its host also requires a profound reprogramming of the fungal transcriptome. Previous studies have been centered on the isolation of minute amounts of RNA from host tissues and the use of microarrays or RNAsequencing (Cairns et al., 2010; Liu et al., 2015). Amorim-Vaz and Sanglard discuss two emerging technologies to improve the capture of fungal RNA and discuss their pros and cons, and how microbial transcriptomics can benefit from them (Amorim-Vaz and Sanglard). The underrepresentation of fungal DNA has also been hampering the precise characterization of the fungal communities in the human host, their composition and dynamics, and contribution to disease. By examining the human gut mycobiota, Strati et al. highlight important implications of age- and gender-dependent interindividual variation in microbiota diversity, and consequently susceptibility to fungal infection (Strati et al.).

\section{REFERENCES}

Bezerra, A. R., Simoes, J., Lee, W., Rung, J., Weil, T., Gut, I. G., et al. (2013). Reversion of a fungal genetic code alteration links proteome instability with
Systems biology has demonstrated that the fungal cell wall is a highly dynamic organelle (Brown et al., 2015). Based on the proteomics of fungal extracellular vesicles (EVs), Nimrichter et al. discuss the contributions of EVs to the interaction with host cells (Nimrichter et al.). Likewise, fungal sphingolipids form a unique and complex group of bioactive lipids with a role in microbial pathogenesis (Bryan et al., 2015). Singh and Del Poeta provide an overview of the methods employed in qualitative and quantitative fungal sphingolipidomics (Singh and Del Poeta). The characterization of the cell wall composition and dynamics is therefore expected to deliver novel therapeutic and vaccination targets (Carvalho et al., 2012a). On the other hand, Prado et al. demonstrate that Paracoccidioides lutzii undergoes a global metabolic adaptation in response to the antifungal argentilactone (Prado et al.). Thus, the use of omics may extend beyond therapeutic target identification to the evaluation of the course of action of antifungals and mechanisms of resistance.

Among fungi, the biology of $C$. albicans is unique due to the flexible reassignment of the leucine CUG codon to serine and synthesis of statistical proteins (Gomes et al., 2007). This aminoacid misincorporation shapes cell surface composition (Miranda et al., 2013) and drives vast phenotypic diversity concerning metabolism, drug resistance and host immunity (Bezerra et al., 2013). Simões et al. resequenced the genome of mistranslating strains to infer that expression of serine tRNAs was linked to mutations in the deneddylase gene (Simões et al.). Besides demonstrating neddylation as a key mechanism in the tolerance to codon ambiguity, this posttranslational modification is highlighted as a promising therapeutic target.

In conclusion, the articles presented here provide an overview of the potential for omics based on concrete examples of their application. With the unrelenting advances in technology, a major contribution of omics to the elucidation of fungal pathogenesis are anticipated, providing crucial information bridging basic research to the patient's bedside.

\section{AUTHOR CONTRIBUTIONS}

$\mathrm{AC}$ and GG conceived and designed the work, drafted the article, and provided final approval of the version to be published.

\section{FUNDING}

This work was supported by the Northern Portugal Regional Operational Programme (NORTE 2020), under the Portugal 2020 Partnership Agreement, through the European Regional Development Fund (FEDER) (NORTE-01-0145-FEDER000013), Fundação para a Ciência e Tecnologia (FCT) (IF/00735/2014 to AC), Conselho Nacional de Desenvolvimento Científico e Tecnológico (CNPq), Brazil, and Fundação de Amparo a Pesquisa do Estado de São Paulo (FAPESP), Brazil.

genomic and phenotypic diversification. Proc. Natl. Acad. Sci. U.S.A. 110, 11079-11084. doi: 10.1073/pnas.1302094110

Bochud, P. Y., Chien, J. W., Marr, K. A., Leisenring, W. M., Upton, A., Janer, M., et al. (2008). Toll-like receptor 4 polymorphisms and aspergillosis in stem-cell 
transplantation. N. Engl. J. Med. 359, 1766-1777. doi: 10.1056/NEJMoa08 02629

Brown, L., Wolf, J. M., Prados-Rosales, R., and Casadevall, A. (2015). Through the wall: extracellular vesicles in Gram-positive bacteria, mycobacteria and fungi. Nat. Rev. Microbiol. 13, 620-630. doi: 10.1038/nrmicro3480

Brunke, S., Quintin, J., Kasper, L., Jacobsen, I. D., Richter, M. E., Hiller, E., et al. (2015). Of mice, flies-and men? Comparing fungal infection models for largescale screening efforts. Dis. Model. Mech. 8, 473-486. doi: 10.1242/dmm.019901

Bryan, A. M., Del Poeta, M., and Luberto, C. (2015). Sphingolipids as regulators of the phagocytic response to fungal infections. Mediators Inflamm. 2015:640540. doi: $10.1155 / 2015 / 640540$

Cairns, T., Minuzzi, F., and Bignell, E. (2010). The host-infecting fungal transcriptome. FEMS Microbiol. Lett. 307, 1-11. doi: 10.1111/j.1574-6968. 2010.01961.x

Carvalho, A., Cunha, C., Iannitti, R. G., Casagrande, A., Bistoni, F., Aversa, F., et al. (2012a). Host defense pathways against fungi: the basis for vaccines and immunotherapy. Front. Microbiol. 3:176. doi: 10.3389/fmicb.2012.00176

Carvalho, A., Cunha, C., Pasqualotto, A. C., Pitzurra, L., Denning, D. W., and Romani, L. (2010). Genetic variability of innate immunity impacts human susceptibility to fungal diseases. Int. J. Infect. Dis. 14, e460-e468. doi: $10.1016 /$ j.ijid.2009.06.028

Carvalho, A., De Luca, A., Bozza, S., Cunha, C., D’Angelo, C., Moretti, S., et al. (2012b). TLR3 essentially promotes protective class I-restricted memory $\mathrm{CD}^{+}{ }^{+} \mathrm{T}$-cell responses to Aspergillus fumigatus in hematopoietic transplanted patients. Blood 119, 967-977. doi: 10.1182/blood-2011-06-362582

Carvalho, A., Pasqualotto, A. C., Pitzurra, L., Romani, L., Denning, D. W., and Rodrigues, F. (2008). Polymorphisms in toll-like receptor genes and susceptibility to pulmonary aspergillosis. J. Infect. Dis. 197, 618-621. doi: $10.1086 / 526500$

Cheng, S. C., Quintin, J., Cramer, R. A., Shepardson, K. M., Saeed, S., Kumar, V., et al. (2014). mTOR- and HIF-1alpha-mediated aerobic glycolysis as metabolic basis for trained immunity. Science 345:1250684. doi: 10.1126/science.1250684

Cheng, S. C., Scicluna, B. P., Arts, R. J., Gresnigt, M. S., Lachmandas, E., Giamarellos-Bourboulis, E. J., et al. (2016). Broad defects in the energy metabolism of leukocytes underlie immunoparalysis in sepsis. Nat. Immunol. 17, 406-413. doi: 10.1038/ni.3398

Cunha, C., Aversa, F., Lacerda, J. F., Busca, A., Kurzai, O., Grube, M., et al. (2014). Genetic PTX3 deficiency and aspergillosis in stem-cell transplantation. N. Engl. J. Med. 370, 421-432. doi: 10.1056/NEJMoa1211161

Cunha, C., Aversa, F., Romani, L., and Carvalho, A. (2013). Human genetic susceptibility to invasive aspergillosis. PLoS Pathog. 9:e1003434. doi: 10.1371/journal.ppat.1003434

Cunha, C., and Carvalho, A. (2012). Host genetics and invasive fungal diseases: towards improved diagnosis and therapy? Expert Rev. Anti Infect. Ther. 10, 257-259. doi: 10.1586/eri.12.3

Cunha, C., Carvalho, A., Esposito, A., Bistoni, F., and Romani, L. (2012). DAMP signaling in fungal infections and diseases. Front. Immunol. 3:286. doi: $10.3389 /$ fimmu.2012.00286

Cunha, C., Di Ianni, M., Bozza, S., Giovannini, G., Zagarella, S., Zelante, T., et al. (2010). Dectin-1 Y238X polymorphism associates with susceptibility to invasive aspergillosis in hematopoietic transplantation through impairment of both recipient- and donor-dependent mechanisms of antifungal immunity. Blood 116, 5394-5402. doi: 10.1182/blood-2010-04-279307

Cunha, C., Giovannini, G., Pierini, A., Bell, A. S., Sorci, G., Riuzzi, F., et al. (2011). Genetically-determined hyperfunction of the S100B/RAGE axis is a risk factor for aspergillosis in stem cell transplant recipients. PLoS ONE 6:e27962. doi: 10.1371/journal.pone.0027962

Cunha, C., Monteiro, A. A., Oliveira-Coelho, A., Kuhne, J., Rodrigues, F., Sasaki, S. D., et al. (2015). PTX3-based genetic testing for risk of aspergillosis after lung transplant. Clin. Infect. Dis. 61, 1893-1894. doi: 10.1093/cid/civ679

Dix, A., Vlaic, S., Guthke, R., and Linde, J. (2016). Use of systems biology to decipher host-pathogen interaction networks and predict biomarkers. Clin. Microbiol. Infect. 22, 600-606. doi: 10.1016/j.cmi.2016. 04.014

Gomes, A. C., Miranda, I., Silva, R. M., Moura, G. R., Thomas, B., Akoulitchev, A., et al. (2007). A genetic code alteration generates a proteome of high diversity in the human pathogen Candida albicans. Genome Biol. 8:R206. doi: 10.1186/gb-2007-8-10-r206

Kontoyiannis, D. P., Marr, K. A., Park, B. J., Alexander, B. D., Anaissie, E. J., Walsh, T. J., et al. (2010). Prospective surveillance for invasive fungal infections in hematopoietic stem cell transplant recipients, 2001-2006: overview of the Transplant-Associated Infection Surveillance Network (TRANSNET) Database. Clin. Infect. Dis. 50, 1091-1100. doi: 10.1086/651263

Kumar, V., Cheng, S. C., Johnson, M. D., Smeekens, S. P., Wojtowicz, A., Giamarellos-Bourboulis, E., et al. (2014). Immunochip SNP array identifies novel genetic variants conferring susceptibility to candidaemia. Nat. Commun. 5, 4675. doi: $10.1038 /$ ncomms5675

Liu, Y., Shetty, A. C., Schwartz, J. A., Bradford, L. L., Xu, W., Phan, Q. T., et al. (2015). New signaling pathways govern the host response to C. albicans infection in various niches. Genome Res. 25, 679-689. doi: $10.1101 /$ gr.187427.114

Lupianez, C. B., Canet, L. M., Carvalho, A., Alcazar-Fuoli, L., Springer, J., Lackner, M., et al. (2015). Polymorphisms in host immunity-modulating genes and risk of invasive aspergillosis: results from the AspBIOmics consortium. Infect. Immun. 84, 643-657. doi: 10.1128/IAI.01359-15

Miranda, I., Silva-Dias, A., Rocha, R., Teixeira-Santos, R., Coelho, C., Goncalves, T., et al. (2013). Candida albicans CUG mistranslation is a mechanism to create cell surface variation. MBio 4:e00285-13. doi: 10.1128/mBio.00285-13

Netea, M. G., Joosten, L. A., Latz, E., Mills, K. H., Natoli, G., Stunnenberg, H. G., et al. (2016). Trained immunity: a program of innate immune memory in health and disease. Science 352:aaf1098. doi: 10.1126/science.aaf1098

Oliveira-Coelho, A., Rodrigues, F., Campos, A. Jr., Lacerda, J. F., Carvalho, A., and Cunha, C. (2015). Paving the way for predictive diagnostics and personalized treatment of invasive aspergillosis. Front. Microbiol. 6:411. doi: 10.3389/fmicb.2015.00411

Pagano, L., Caira, M., Candoni, A., Offidani, M., Martino, B., Specchia, G., et al. (2010). Invasive aspergillosis in patients with acute myeloid leukemia: a SEIFEM-2008 registry study. Haematologica 95, 644-650. doi: 10.3324/haematol.2009.012054

Saeed, S., Quintin, J., Kerstens, H. H., Rao, N. A., Aghajanirefah, A., Matarese, F., et al. (2014). Epigenetic programming of monocyte-tomacrophage differentiation and trained innate immunity. Science 345:1251086. doi: 10.1126/science. 1251086

Smeekens, S. P., Ng, A., Kumar, V., Johnson, M. D., Plantinga, T. S., Van Diemen, C., et al. (2013a). Functional genomics identifies type I interferon pathway as central for host defense against Candida albicans. Nat. Commun. 4, 1342. doi: $10.1038 /$ ncomms 2343

Smeekens, S. P., Van De Veerdonk, F. L., Kullberg, B. J., and Netea, M. G. (2013b). Genetic susceptibility to Candida infections. EMBO Mol. Med. 5, 805-813. doi: $10.1002 / \mathrm{emmm} .201201678$

Wojtowicz, A., Lecompte, T. D., Bibert, S., Manuel, O., Rueger, S., Berger, C., et al. (2015). PTX3 polymorphisms and invasive mold infections after solid organ transplant. Clin. Infect. Dis. 61, 619-622. doi: 10.1093/cid/civ386

Conflict of Interest Statement: The authors declare that the research was conducted in the absence of any commercial or financial relationships that could be construed as a potential conflict of interest.

Copyright $(\odot) 2017$ Carvalho and Goldman. This is an open-access article distributed under the terms of the Creative Commons Attribution License (CC BY). The use, distribution or reproduction in other forums is permitted, provided the original author(s) or licensor are credited and that the original publication in this journal is cited, in accordance with accepted academic practice. No use, distribution or reproduction is permitted which does not comply with these terms. 